\title{
EXERGAMES: USO PEDAGÓGICO E SUAS BASES TEÓRICAS NA EDUCAÇÃO
}

\author{
Fernanda C. A. O. FRONZA ${ }^{1}$ \\ Fernando Luiz CARDOSO ${ }^{2}$
}

Resumo: Os exergames são tecnologias digitais dirigidas a atividade física que tem sido utilizado mundialmente em escolas de maneira lúdica ou pedagógica. $\mathrm{O}$ objetivo deste artigo é identificar e caracterizar os estudos que usam exergames na escola, apontar quais as potencialidades pedagógicas destes jogos para a educação e se suas bases teóricas estão consolidadas para seu uso escolar. O estudo é uma revisão sistemática em bases importantes na área de educação e saúde com os descritores exergames e educação em inglês. Foram selecionados 4 artigos, com sujeitos de pesquisa constituídos por crianças e professores de escolas, de cunho empírico porém nem todos seguiram protocolos específicos de avaliação. A metade deles se respaldou em alguma teoria para justificar seus achados, e as contribuições foram relevantes tanto para alunos e professores a partir da vivência empírica com o uso dos exergames na escola. Concluise que as respostas físicas e comportamentais dos estudantes nos jogos estudados foram significativas nas escolas, no entanto os estudos avaliados não se basearam em quaisquer teorias consolidadas da área.

Palavras-chave: Exergames. Videogames. Educação. Escola.

\section{Introdução}

O jogo é uma das manifestações mais antigas da cultura, sendo inerente ao comportamento dos animais, revelando-se mais do que um fenômeno fisiológico ou um reflexo psicológico. A realidade do jogo ultrapassa a esfera da vida humana por isso não se baseia em qualquer elemento racional, dado que não é privilégio apenas espécie humana, e a sua existência não está ligada a qualquer grau determinado de civilização. De todas as teorias que buscam explicar a função biológica do jogo partem do pressuposto de que este se encontra ligada alguma coisa que não seja o próprio jogo, algo descrito como uma espécie de finalidade biológica ou evolutiva (HUIZINGA, 2000).

\footnotetext{
${ }^{1}$ Doutoranda em Educação. UDESC - Universidade do Estado de Santa Catarina. Centro de Ciências Humanas e da Educação - Pós-graduação em Educação. Florianópolis - SC - Brasil. 88035-001 fernandacerveira@yahoo.com.br

${ }^{2}$ Doutor em Sexualidade Humana pelo Institute for Advanced Study in Human Sexuality. Professor Titular junto ao Programa de Pós-Graduação em Ciência do Movimento Humano e do Programa de PósGraduação em Educação da UDESC. Florianópolis - SC - Brasil. 88080-350 fernandocardoso.ph.d.lagesc@gmail.com
} 
Dada a suas origens tão antigas, o ato de jogar se modifica e se reinventa com o passar do tempo e, atualmente, à luz da tecnologia disponível de sensoriamento e rastreio, uma nova forma ou classe de jogos surgiu, combinando videogame e exercício físico. Os Exergames ou EXG são definidos como tecnologias dirigidas a atividades físicas, como os jogos de vídeo game, que desenvolvem uma proposta lúdica, exigindo que o usuário seja fisicamente ativo ou se exercite para poder jogar. Estes jogos pressupõem que o jogador execute movimentos que abrangem habilidades motoras finas até ações com o corpo inteiro para participar de esportes virtuais, exercícios de fitness ou outras atividades físicas interativas propostas pelos jogos (VAGHETT; MUSTARO; BOTELHO, 2011; ACSM, 2013).

Os exergames surgem então da fusão dos jogos de vídeo com aparelhos de ginástica, ganhando força a partir de meados de 2006 quando se observou um crescente interesse pela atividade. Um dos argumentos mais consistentes para a ênfase nestes jogos foi a preocupação com os níveis elevados de obesidade (especialmente em crianças) em sociedades ocidentais. Assim, este tipo de tecnologia, que é lúdica e bem aceita pelas crianças, poderia ser aproveitada de forma a envolvê-las em atividade física por um maior período de tempo. Atrelada a esta questão, aparecem também como justificativa para o aumento da visibilidade em exergames, o fato de representar um enorme mercado como potencial para o lucro e por adotar recursos que facilitam a sua prática como controladores sensíveis ao movimento, sendo o Nintendo Wii o precussor destes dispositivos (SINCLAIR; HINGSTON; MASEK, 2007).

Neste contexto, o jogo é algo inerente ao homem e está presente em diferentes instituições educativas formais e informais. Os exergames são um tipo de jogos, com características bem peculiares, que têm sido usados como uma ferramenta pedagógica para a educação presencial ou não presencial, em função da sua capacidade de entretenimento e por possibilitar a aquisição de um estilo de vida saudável (HUIZNIGA, 2000; NADLER, 2008). Sua possibilidade de incorporá-los no currículo tem atraído a atenção dos educadores em escolas e universidades, e estudos experimentais já desenvolvidos avaliaram o impacto do uso de jogos em disciplinas tais como matemática, ciências, linguagem e ciência da computação. Os achados indicam que jogar exegames na sala de aula ou em atividades curriculares obtêm-se resultados positivos em termos de motivação dos alunos e eficácia na aprendizagem em sala Além disso, o jogo é o grande conteúdo ministrado pela educação física, permitindo inserção de forma lúdica de temáticas como os esportes, as lutas, as danças, as ginásticas e até 
mesmo ensinar o jogo com o próprio jogo (PAPASTERGIOU, 2009; VAGHETTI; MUSTARO; BOTELHO, 2011).

Os exergames representam não apenas a possibilidade de uma ferramenta pedagógica no currículo da Educação Física, mas também configuram-se como o próprio conteúdo da cultura digital a ser socializado, dando visibilidade ao surgimento de novos modos de cognição e de percepção, permitindo a configuração de novas práticas curriculares pautadas em diretrizes pedagógicas construídas no século XIX (VAGHETTI; MUSTARO; BOTELHO, 2011).Diante disto, o objetivo deste estudo foi investigar dentro da produção literária quais as características dos estudos sobre exergames em escolas, quais as evidências científicas sobre as potencialidades pedagógicas destes jogos para a área da educação e ainda, se suas bases teóricas estão consolidadas para serem usados em escolas?

\section{Método}

O estudo foi desenvolvido através de uma revisão sistemática de literatura com vistas a explicitar os principais achados de pesquisas sobre o tema em questão com um olhar qualitativo sobre a análise do material. A busca de dados foi realizada nas bases de dados importantes em ciências humanas e da saúde, buscando rastreamento de artigos com os descritores exergames e educação, conectadas pelo o operador booleano AND. Assim, as buscas ocorreram no mês de novembro de 2011 nas bases Science Direct, Pubmed, ERIC (Educational Resources Information Center), REdAlyc (Red de Revistas Científicas de América Latina y el Caribe, España y Portugal).

Os critérios de elegibilidade para integrar o estudo exigiram que os artigos abordassem pesquisas com exergames na educação básica, pois este trariam experiências de escolas e seriam de caráter empírico; ser escrito em língua inglesa ou português; não ser estudo de revisão.

A partir dos critérios adotados, foram selecionados 2estudos na base de dados Science Direct, 1 na SciELO,1 na PubMed, 1 na ERIC. Após leitura detalhada dos resumos, obteve-seuma amostra deartigos (Figura 1) que atendiam aos pressupostos deste estudo, sendo excluídas as duplicidades. 


\section{Resultados}

Os resultados são apresentados em função do fluxo dos artigos expostos no quadro 1, bem como na avaliação qualitativa dos achados dos estudos categorizadas de modo a atender os objetivos em questão.

Quadro 1 - Fluxograma dos artigos elegíveis para o estudo

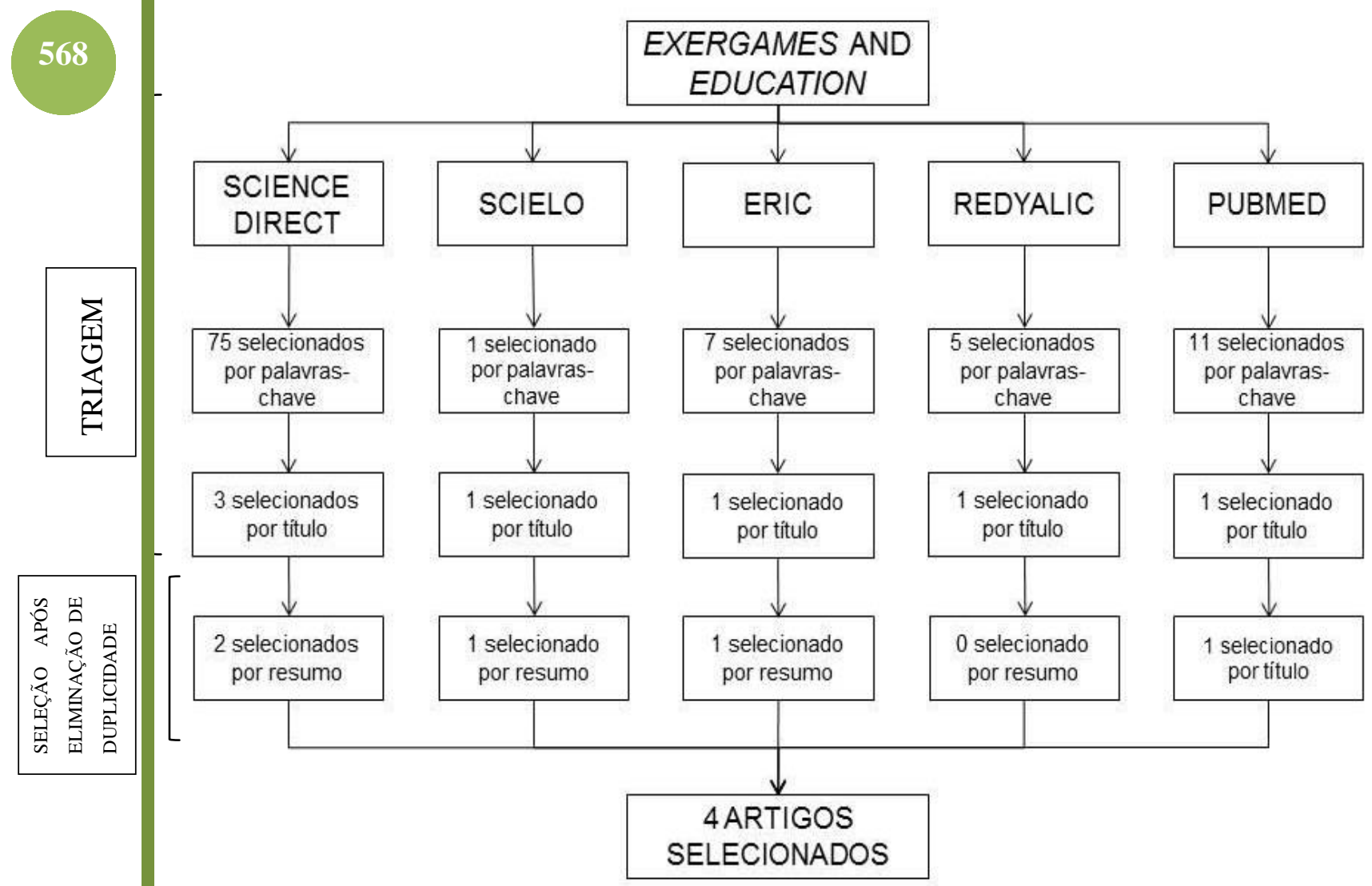

Fonte: Elaboração própria.

O quadro 1 demonstra os aspectos relevantes das produções científicas com o tema dos exergames na educação. Da análise das informações, observa-se que as produções são recentes (2011-2013), com sujeitos de pesquisa que variam de alunos até os próprios docentes. Os objetivos, de modo geral, tratam de investigar a eficácia dos exergames para em experiências levadas para a salada de aula ou educação física escolar, portanto todos os estudos selecionados tem um caráter empírico, embora nem todos apliquem algum protocolo para os jogos. Ao avaliar as questões teóricas que pudessem embasar os estudos, percebeu-se que apenas dois se basearam em alguma teoria consolidada. As contribuições dos estudos destacam os exergames enquanto recurso pedagógico amigável de aproximação dos alunos nas escolas. 
Quadro 1 - Comparação dos estudos envolvendo exergames na educação

\begin{tabular}{|c|c|c|c|c|c|c|c|c|c|}
\hline RASE & ARI & o & $\underset{\text { AWO }}{\text { AUTOR E }}$ & OBEEIMO & POPULAC̄o & IPO DE PESOUISA & $\begin{array}{l}\text { APUCACÃO } \\
\text { DE } \\
\text { PROTOCOLO }\end{array}$ & BASE IEÓraca & COAIrabaçōEs \\
\hline ERAC & $\begin{array}{l}\text { Playing Ex: } \\
\text { the Chassi } \\
\text { service T } \\
\text { Motivation, } \\
\text { Ellot, and P }\end{array}$ & $\begin{array}{l}\text { games in } \\
\text { mon:Pre- } \\
\text { chers' } \\
\text { assion, } \\
\text { Ispectives }\end{array}$ & $\begin{array}{l}\text { Lin Lin e } \\
\text { Zhang } \\
\text { (2011) }\end{array}$ & 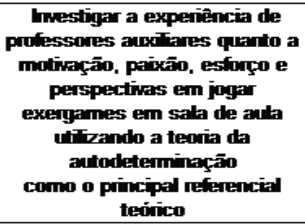 & $\begin{array}{c}140 \text { putessones } \\
\text { da educaçāo } \\
\text { bérsica de } 18 \text { a } \\
23 \text { anos }\end{array}$ & 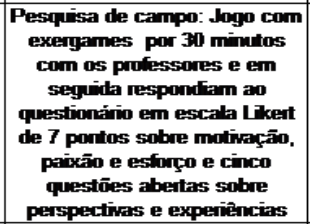 & $\sin$ & 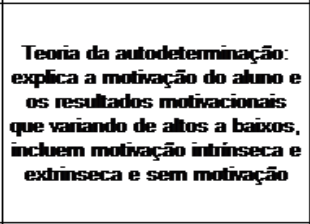 & 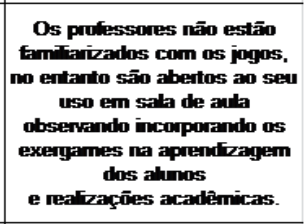 \\
\hline \multirow{2}{*}{$\begin{array}{l}\text { Science } \\
\text { Diect }\end{array}$} & $\begin{array}{r}\text { Impact of ex } \\
\text { physical a: } \\
\text { mativa: } \\
\text { Gementa: } \\
\text { students: } \\
\text { stu }\end{array}$ & $\begin{array}{l}\text { yames on } \\
\text { nily and } \\
\text { sch } \\
\text { schood } \\
\text { follow-up }\end{array}$ & Sim (2013) & 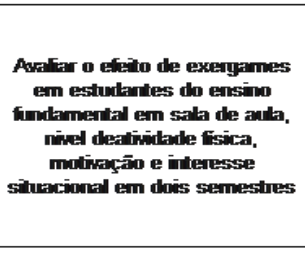 & $\begin{array}{c}70 \text { paricipantes } \\
\text { de } 9 \text { a } 12 \text { anos } \\
\text { (30) meninos e } \\
40 \text { meninas) }\end{array}$ & $\begin{array}{c}\text { Pesquisa de campo: Estudo } \\
\text { unginumbal de intervençäo } \\
\text { com estudantes de uma } \\
\text { escola elementar dos Estados } \\
\text { Unidos }\end{array}$ & Nลด & Näo se aplica & 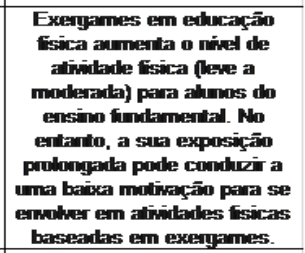 \\
\hline & $\begin{array}{l}\text { The ef: } \\
\text { exergames- } \\
\text { physical } \\
\text { lessons in }\end{array}$ & $\begin{array}{l}\text { cy of } \\
\text { somporated } \\
\text { ucation } \\
\text { meancingy }\end{array}$ & $\begin{array}{l}\text { Lwin e } \\
\text { Malik } \\
\text { (2012) }\end{array}$ & 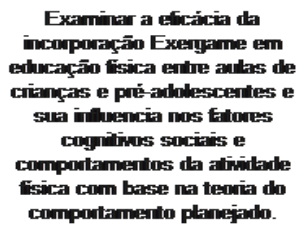 & \begin{tabular}{|c|}
112 \\
panticipantes de \\
10 a 12 anos de \\
5 e 6 série
\end{tabular} & 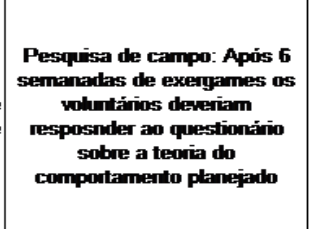 & $\sin$ & 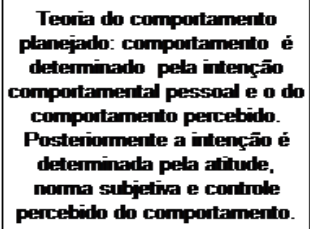 & 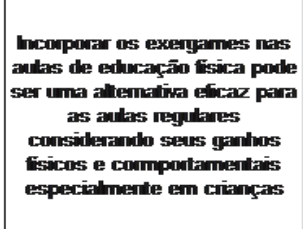 \\
\hline Sciebo & $\begin{array}{l}\text { Os exery: } \\
\text { educacaso fis: } \\
\text { na cultur }\end{array}$ & $\begin{array}{l}\text { mes e a } \\
\text { a escolar } \\
\text { digital }\end{array}$ & $\begin{array}{c}\text { Baracho et } \\
\text { al (2012) }\end{array}$ & 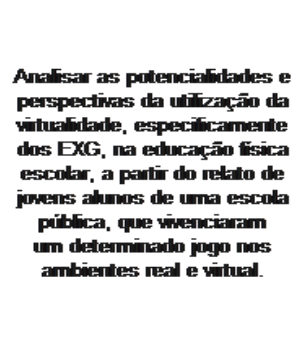 & $\begin{array}{c}117 \text { jowens de } \\
\text { ambos os } \\
\text { sexos com } \\
\text { intade entre } 13 \mathrm{e} \\
14 \text { anos }\end{array}$ & 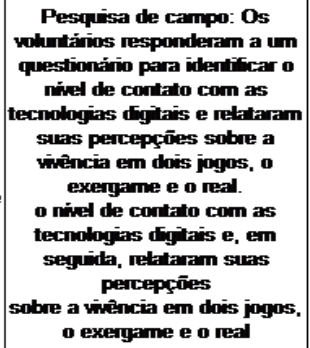 & Nลลอ & Nลื̄o se aplica & 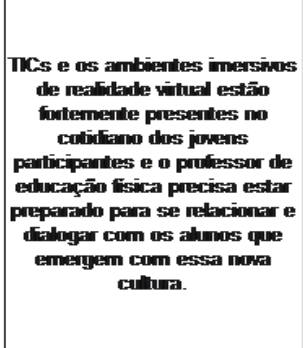 \\
\hline & & & & & & & & & \\
\hline
\end{tabular}

Fonte: Elaboração própria.

\section{Discussão}

A discussão será conduzida em três momentos segundo as análises realizadas, sendo o primeiro a caracterização dos estudos dentro das produções científicas rastreadas sobre a temática em questão.

Observando-se o número de artigos encontrados após aplicação dos critérios de seleção envolvendo exergames e a atualidade daqueles que foram encontrados, entendese que as produções científicas, abordando esta temática, são consideradas atuais no cenário acadêmico em função do recente crescimento e popularização destas tecnologias. A mudança de paradigma entre o que estava sendo produzido em termos de dispositivos e jogos de vide-games aconteceu a partir do ano de 2006, quando a Nintendo lançou o Nintendo Wii, cujo console era construído a partir de sensores de movimento que permitia o corpo do jogador interagir com o game quando em movimento (SINCLAIR; HINGSTON; MASEK, 2007; ARAÚJO et al., 2011).A partir 
de então as perspectivas entre uso e usuário de games foram ampliadas e alcançou contornos além daqueles puramente lúdicos em um público de crianças a idosos.

Esses fenômenos alavancados pelo avanço tecnológico e mercadológico, como no caso dos exergames, segundo Lévy (1999), são conhecidos como cibercultura e ciberespaço:

O termo [ciberespaço] especifica não apenas a infraestrutura material da comunicação digital, mas também o universo oceânico de informação que ela abriga, assim como os seres humanos que navegam e alimentam esse universo. Quanto ao neologismo 'cibercultura', especifica aqui o conjunto de técnicas (materiais e intelectuais), de práticas, de atitudes, de modos de pensamento e de valores que se desenvolvem juntamente com o crescimento do ciberespaço. (LÉVY, 1999, p. 17).

Estes conceitos estão imbricados e se adequam à atualidade em que se vive. A cibercultura encontra-se ligada ao virtual, representado pelos jogos, seja de forma direta (através da digitalização da informação que pode ser aproximada da virtualização) ou indireta (encorajada pelo estilo independente de lugar geográfico ou de tempo/assíncrona).

Ainda na caracterização, os sujeitos das pesquisas avaliadas, com exceção do estudo de Lin e Zanhg (2001) que trabalha com professores, são crianças com idade a de 9 a 14 anos, sugerindo que as outras faixas etárias mais jovens não foram testadas ainda na escola. De acordo com Piaget (2007), em seus estudos sobre a epistemologia genética, a inteligência é uma adaptação constante, e a aquisição de conhecimentos, independentemente do estágio em que os seres humanos se encontram, acontece por meio da relação sujeito/objeto, sendo esta relação dialética por processos de assimilação, acomodação e equilibração. Neste contexto, entende-se que as crianças, independentemente do estágio cognitivo que estejam vivenciando, possuem condições plenas de desenvolvimento pedagógico a partir dos estímulos e desafios que os jogos propõem, respeitado o grau de dificuldade que as mesmas consigam acompanhar.

Por fim, os estudos apresentados tiveram uma natureza empírica de campo, no entanto, apenas a metade deles apresentou algum protocolo para a análise criteriosa dos exergames. Infelizmente nenhum dos estudos fez qualquer inferência em termos teóricos ou conceituais sobre a finalidade ou fundamentos dos jogos. Para Popper (1972), todas as ciências são passíveis de testagem e o conceito de falseabilidade, corresponde ao o critério de avaliar a cientificidade de uma teoria, com base em procedimentos críticos, na medida em que ela estabelece hipóteses podendo ser aceita 
ou refutada com base na experiência. Assim, testar a eficácia de um recurso requer procedimentos rigorosos para a sua reprodução e confiabilidade dos seus resultados.

Sobre a questão do uso de exergames nas escolas, buscou-se nos estudos analisados identificar quais as potencialidades pedagógicas destes jogos para a educação formal. Desta maneira, os artigos selecionados que tratam dos exergames na escola têm buscado investigar estes games como ferramenta acadêmica, buscando diferentes objetivos dentro de uma proposta empírica. Inclusive um dos estudos (LIN; ZANHG, 2001) se propõe a trabalhar com percepções e sensações dos professores sobre os jogos, considerando que estes são os responsáveis por incluí-los como instrumento pedagógico para os alunos dentro do seu planejamento de aula.

Sobre esta questão, Ramos (2008) ressalta ouso dos jogos eletrônicos como recurso pedagógico e destaca o potencial dos seus reflexos sobre o desenvolvimento humano. Desta maneira, o professor passa ater uma função estratégica na escola: precisa incorporar este tipo de jogo em sua prática pedagógica para promover a aprendizagem dos alunos e ainda, conhecer e interagir com esta nova geração que chega à sala de aula.

As contribuições dos estudos são favoráveis em relação à inclusão dos exergames nas práticas escolares, sinalizando tanto a predisposição do docente, considerando estes jogos como ferramentas pedagógicas, quanto a aceitação dos alunos que se apresentam motivados tendo resultados testados em relação a ganhos físicos e comportamentais. Ademais, essas tecnologias estão fortemente associadas ao cotidiano desta geração de crianças e adolescentes, o que facilita a adesão destas práticas em ambiente escolar. Para Vaghetti et al. (2013) a educação física escolar precisa ser repensar a sua prática que, em sua maioria, é pautada no ensino esportes coletivos, privilegiando os alunos com habilidades para os esportes com bola. Entretanto, quando esta linha é seguida ,o grande conteúdo da Educação Física que são os jogos - que contemplaria de forma lúdica os esportes, as lutas, as danças, as ginásticas e o próprio jogo - ficam em segundo plano. Os exergames representam mais uma aplicação dos jogos e podem ser usados como ambientes virtuais de aprendizagem para o ensino de Educação Física escolar, iniciação esportiva e o ensino de esportes, bem como estarem inseridos ensino superior na demonstração de conteúdos de disciplinas como Fisiologia do Exercício, Biomecânica e Aprendizagem Motora.

O último aspecto da análise está pautado no uso de jogos como os exergames com fins didáticos e educativos, o que pressupõe um embasamento teórico alicerçado em bases consistentes. No presente estudo, a metade dos artigos não apresenta uma base 
teórica para trabalhar estes jogos como recurso educacional ou qualquer tipo de descrição ou conceituação, e os demais apoiam seus achados em teorias da psicologia baseada em questões comportamentais, como na teoria do comportamento planejado para Lwin e Malik (2012) e na autodeterminação como fazem Lin e Zanhg (2001).

No entanto, uma das teorias mais clássicas usada em jogos e que tem aplicação em diferentes domínios como no esporte, educação e em games é a teoria do fluxo, desenvolvida por Csikszentmihalyi em nos anos 70 e em nenhum momento foi citada. Esta teoria pressupõe que qualquer ideia de atratividade em um jogo é dependente da forma como a habilidade do jogador é combinada com os desafios e com a narrativa do jogo. Segundo a teoria de Csikszentmihalyi, durante a experiência de fluxo que acontece, por exemplo, quando se joga um game com características e sensações tão reais como os exergames, o indivíduo é absorvido por um estado mental em que se perde a noção do tempo e as preocupações, o nível de foco aumenta, bem como o desempenho e sensação de prazer da atividade (SINCLAIR et al., 2007). Por se tratar de uma teoria aplicada à realidade dos jogos representados pelos exergames, a teoria do fluxo parece representar um universo epistemológico com um respaldo sólido a ser melhor explorado pelos estudos nesta área.

Nesse contexto acredita-se que o amadurecimento da área em termos psicométricos possa a começar a responder perguntas importantes que os simpatizantes dos exergames como um instrumento pedagógico provavelmente possuem, como por exemplo: a. qual é o conteúdo dos exergames? b. como devem ser aplicados em ambiente escolar para crianças e adolescentes de distintas idades? c. quais potencialidades humanas podem vir a desenvolver? d. que aspectos do processo de ensino aprendizagem podem facilitar?

\section{Conclusão}

O cenário tecnológico atual oportuniza recursos com uma multiplicidade de significados, dentre os quais se destacam os exergames, que incluem na sua proposta aspectos de lazer e entretenimento, motores e educativos. Os estudos são recentes e tem investigado estes jogos em uma perspectiva empírica nas escolas, encontrando respostas físicas e comportamentais meramente descritivas, norteando a aproximação deste recurso como ferramenta pedagógica.

No entanto, o avanço desta temática ainda não está acompanhado de um referencial com uma base teórica e metodológica consolidada e dirigida aos exergames 
na escola, pois dos estudos encontrados apenas a metade utilizou-se de alguma teoria que pudesse descrever, interpretar ou explicar o fenômeno. Além disso, nenhum estudo que analise o conteúdo, as dimensões e funções dos exergames foi encontrado. Sabe-se que a área dos jogos ainda está em estruturação, e por isso, ressalta-se a importância de estudos com cunho tanto epistemológico que melhor descrevam e conceitue o fenômeno, como também, estudos de cunho metodológico empírico que identifique e mensure as importantes qualidades desse fenômeno. Só então poderemos iniciar uma nova geração de pesquisas sobre os exergames com modelos de pesquisa que não apenas os descrevam, mas também, os testem em termos de validade e confiabilidade. Isto é, o que desenvolvem ou para que servem e se funcionam bem como instrumento pedagógico?

\title{
EXERGAMES: EDUCATIONAL USE AND THEORETICAL BASES IN EDUCATION
}

\begin{abstract}
Exergames are digital technologies produced to physical activity that is used world wide in schools for educational or playful way purposes. The objective of the study is to characterize the studies using exergames in schools, identifying the pedagogical potential of these games for education field and if its theoretical bases are consolidated for school use. The study is a systematic review of important bases in education and health using exergames and education as descriptors in English. There were a total of 4 papers, with research subjects consisting of children and school teachers, with an empirical nature but not all of them followed specific protocols of analyses. Half of them backed in to a theory to justify their outcomes, and contributions were significant for both students and teachers from the empirical experience with the use of exergames in school. It can conclude that the physical and behavioral game's responses were relevant in studied schools, however studies have not been based on a consolidated theories in the field.
\end{abstract}

Key words: Exergames. Videogames. Education. School.

\section{REFERÊNCIAS}

AMERICAN COLLEGE OF SPORTS MEDICINE. [ACSM]. Information on Exergaming. ACSM Short comunication, 2013. Disponível em:

<https://www.acsm.org/docs/brochures/exergaming.pdf?sfvrsn=6>. Acesso em: 21 jun. 2016.

ARAÚJO, B. M. R. et al. Virtualização esportiva e os novos paradigmas para o movimento humano. Motriz, Rio Claro, v. 17, n. 4, p. 600-609, 2001. 
HUIZINGA, J. Homo Luddens: o jogo como elemento da cultura. 4. ed. São Paulo: Perspectiva, 2000.

LÉVY, P. Cibercultura. São Paulo: 34, 1999.

LIN, L.; ZHANG, T. Playing exergames in the classroom: pre-service teachers' motivation, passion, effort, and perspectives. Journal of Technology and Teacher Education, [Waynesville], v. 19, n. 3, p. 243-260, 2011. Disponível em: <http://eric.ed.gov/?id=EJ944271>. Acesso em: 15 jun. 2016.

LWIN, M.; MALIK, S. The efficacy of exergames-incorporated physical education lessons in influencing drivers of physical activity: a comparison of children and preadolescents. Psychology of Sport and Exercise, [Amsterdã], v. 13, p. 756-760, 2012.

NADLER, D. Exergaming: cardiovascular fitness in immersive virtual environments. Learning \& Leading with Technology, Eugene, v. 35, p. 28-29, 2008. Disponível em: <http://isd194cms.demo.ties.k12.mn.us/sites/bebc19ef-3123-4a78-9c70cc494b7896e6/uploads/resources_from_iste.pdf>. Acesso em: 15 jun. 2016.

PAPASTERGIOU, M. Digital game-based learning in high-school computer science education: impact on educational effectiveness and student motivation. Computers and Education, New York, v. 52, n. 1, p. 1-12, 2009.

PIAGET, J. Epistemologia genética. 3. ed. São Paulo: Martins Fontes, 2007.

POPPER, K. R. A lógica da pesquisa científica. 6. ed. São Paulo: Cultrix, 1972.

RAMOS, D. K. A escola frente ao fenômeno dos jogos eletrônicos: aspectos morais e éticos. Revista Novas Tecnologias na Educação, Porto Alegre, v. 6, n. 1, p. 1-10, 2008. Disponível em: <http://seer.ufrgs.br/renote/article/view/14512>. Acesso em: 15 jun. 2016.

SINCLAIR, J.; HINGSTON, P.; MASEK, M. Considerations for the design of exergames. In: INTERNATIONAL CONFERENCE ON COMPUTER GRAPHICS AND INTERACTIVE TECHNIQUES IN AUSTRALIA AND SOUTHEAST ASIA. 5., 2007, New York. Proceedings... New York: ACM Digital Library, 2007. p. 289-295. Disponível em: <http://dl.acm.org/citation.cfm?id=1321313>. Acesso em: 15 jun. 2016.

VAGHETTI, C. A. O.; MUSTARO, P. N.; BOTELHO, C. S. S. Exergames no ciberespaço: uma possibilidade para educação física. In: Simpósio Brasileiro De Games E Entretenimento Digital, 10., 2011, Salvador. Proceedings... Salvador: SBC, 2011. Disponível em:

<http://www.sbgames.org/sbgames2011/proceedings/sbgames/papers/cult/full/92287_1. pdf $>$. Acesso em: 07 jun. 2016.

VAGHETTI, C. A. O. et al. Exergames: um desafio à educação física na era da tecnologia. Educação \& Tecnologia, Belo Horizonte, v. 12, p. 1-10, 2013. Disponível em: <http://revistas.utfpr.edu.br/pb/index.php/revedutec-ct/article/view/1547.> Acesso em: 07 jun. 2016. 